\title{
Spectral-Energy Efficiency Tradeoff in Relay-Aided Cellular Networks
}

\author{
Ivan Ku, Cheng-Xiang Wang, Senior Member, IEEE, and John Thompson, Senior Member, IEEE
}

\begin{abstract}
A relay cooperation scheme is proposed for the downlink of multicell multiple-input-multiple-output cellular networks. The relay stations (RSs) will cooperatively transmit the signal replicas of all the user equipments obtained during the broadcast phase. We consider different RS decoding strategies during the broadcast phase and joint relay transmission with different degrees of channel state information (CSI) sharing during the relay phase. We also propose the partial semiorthogonal user selection (PSUS) method designed specifically for relay cooperation. The spectral and energy efficiencies are then evaluated for the relay cooperation scheme. Its cooperative costs for different cooperation levels are also investigated. Simulation results indicate that joint $R S$ decoding outperforms independent RS decoding but a cooperative link with a bit rate of an order of magnitude greater than that achievable by the relay network is required. Compared to the competing user selection methods that require global CSI, the proposed PSUS method utilized for relay phase joint transmission operates at less than half of the cooperative cost while introduces only a slight degradation in system performance.
\end{abstract}

Index Terms-Relay cooperation, multicell MIMO network, spectral efficiency, energy efficiency.

\section{INTRODUCTION}

$\mathbf{R}$ ELAY transmission is a promising technology in Long Term Evolution-Advanced (LTE-A) standards [1]. The two-hop relay transmission [2] is the most well-known transmission protocol and will be the main focus in this work. For a network with more relay stations (RSs), relay spectrum planning [3] is essential to avoid relay interference. Although this improves the link level performance, limited gain is observed at the system level as orthogonal frequency reuse schemes do not efficiently utilize the scarce radio resources. Sharing of relay slots can improve resource utilization but introduces relay interference that has to be mitigated [4]-[7].

Manuscript received November 1, 2012; revised March 22 and July 25, 2013; accepted July 26, 2013. The associate editor coordinating the review of this paper and approving it for publication was G. Yue.

The work in this paper has formed part of the Green Radio Core 5 Research Programme of Mobile VCE. This research has been funded by the industrial members of Mobile VCE and the EPSRC. The authors also acknowledge the support from the RCUK for the UK-China Science Bridges Project: R\&D on (B)4G Wireless Mobile Communications, from the Opening Project of the Key Laboratory of Cognitive Radio and Information Processing (Guilin University of Electronic Technology), Ministry of Education (No.: 2013KF01), and from the National Natural Science Foundation of China (NSFC) (Grant No.: 61210002).

I. Ku and C.-X. Wang are with the Joint Research Institute for Signal and Image Processing, School of Engineering and Physical Sciences, Heriot-Watt University, Edinburgh, EH14 4AS, U.K. (e-mail: \{ccik1, chengxiang.wang\}@hw.ac.uk). C.-X. Wang is the corresponding author.

J. S. Thompson is with the Joint Research Institute for Signal and Image Processing, School of Engineering, University of Edinburgh, Edinburgh, EH9 3JL, U.K. (e-mail: john.thompson@ed.ac.uk).

Digital Object Identifier 10.1109/TWC.2013.092013.121692
Relay interference arises because conventional RSs transmit independently. Recent research in cooperative communication shows that significant throughput improvement is possible when network nodes cooperate with each other [8]- [10]. Following the momentum of coordinated multi-point (CoMP) with cooperation among base stations (BSs) [11], the idea of relay cooperation is beginning to gain renewed interest as compared to the more conventional independent relaying schemes. In relay cooperation, the RSs share cooperative information to perform joint relay transmission to the user equipments (UEs). We differentiate this concept from those commonly referenced in the literature where relay cooperation often means that both the direct and relay transmissions are used by the UE for reliable signal decoding, i.e., receive diversity [12]. The authors in [13] proposed an inter-cell relay cooperation scheme to form the uplink joint precoders. The transmission rate of such a scheme was then evaluated in a linear three-cell topology. Here, a linear topology means that the communication nodes are arranged in a straight line. In [14], the spectral efficiency of a single-cell topology is evaluated for a flexible downlink resource management scheme whereby the RSs cooperate to meet a minimum quality of service $(\mathrm{QoS})$ requirement at the UE. Fractional coded relay cooperation was proposed in [15] whereby each RS offers a fraction of its radio resources to relay the data from its neighboring RSs. The bit error rate (BER) performance of the scheme was then evaluated for a linear topology. In [16] and [17], interference alignment and block diagonalization methods were respectively utilized by the cooperating RSs to decompose the relay channel into several parallel channels to improve multiplexing gain. In [18], relay cooperation was achieved through the implementation of network coding techniques derived from XOR-coding or Reed Solomon coding. The outage probability and the block error rate (BLER) performance were then evaluated for a linear topology. In [19], the authors proposed a scheme to select the best transmit antennas distributed across multiple RSs for simultaneous relay transmissions. The outage capacity of this scheme was evaluated for a linear topology. Again, a linear topology with two BSs, two RSs and two UEs was considered in [20] where relay cooperation was implemented between the two RSs. Different levels of relay cooperation in terms of different degrees of RS decoding in the broadcast phase were explored. During the relay phase, it was assumed that the RSs have access to global channel state information (CSI) of all relay channels and are thus able to jointly design the precoders for joint relay transmission. In [21], an asymmetric relay cooperation scheme was proposed. Its throughput was maximized for a linear topology of one BS, two RSs and two UEs. 
Most works in relay cooperation focused on the spectral efficiency or throughput performance of a system while others solely focused on energy efficiency. For example in [22], energy efficiency improvement was shown by combining relay selection with cooperative relay beamforming for a linear topology. However, given the significance of energy efficiency for future mobile networks [23] where both power and bandwidth constrict the achievable gains, a joint spectral-energy efficiency performance evaluation, e.g., [24] and [25], is imperative. However, both [24] and [25] evaluated the spectral-energy efficiency performance of their proposed relay cooperation schemes using only the radio frequency (RF) transmit power. Circuit power, especially in multipleinput-multiple-output (MIMO) systems, drains a considerable amount of the input power and this necessitates its adoption in energy efficiency evaluation, as given in [26], to provide realistic results. In this work, we propose a relay cooperation scheme for downlink multicell MIMO cellular networks to address some of the shortcomings of the previous schemes. The following summarizes our contributions.

1) Different cooperation levels among the RSs are investigated in our proposed scheme. We consider different RS decoding strategies for the broadcast phase and joint relay transmission with different degrees of CSI sharing for the relay phase. This is different from [20] which only considered several RS decoding strategies for the broadcast phase. We also take into account both the RF transmit power and the circuit power of the system, thus reflecting a more realistic scenario for the overall energy consumption.

2) We extend the work in [20] and [26] to include multiuser diversity gain by proposing a low complexity normbased user selection method for the relay cooperation scheme. It is designed to operate without excessively loading the cooperative links as it is well-known that this is a major limitation for cooperative systems [17]. To the best of our knowledge, our user selection method is new as none of the existing relay cooperation works addressed this issue and most existing methods were designed for the point-to-point MU-MIMO systems, e.g. [27], with the intention of maximizing capacity.

3) We quantify the cost of different cooperation levels in terms of the bit rate needed at the cooperative links to sustain the performance gains that these cooperative strategies provide. The average power consumption of the cooperative links is also given to further emphasize the cooperative costs involved.

Notations: Boldface characters, e.g., $\mathbf{X}$, denote matrices while scalar values are denoted by upper or lower case italics, e.g., $X$ or $x$. The Hermitian transpose, transpose, inverse and Frobenius norm of a matrix are represented by $\mathbf{X}^{\mathrm{H}}, \mathbf{X}^{\mathrm{T}}, \mathbf{X}^{-1}$ and $\|\mathbf{X}\|_{\mathrm{F}}^{2}$, respectively, while $\mathbf{I}_{N}$ is an $N \times N$ identity matrix. The expectation, determinant and ceiling operators are denoted by $\mathbb{E}\{\cdot\}$, det $[\cdot]$ and $\lceil\cdot\rceil$, respectively. The cardinality of set $\mathcal{S}$ is $|\mathcal{S}|$ and $x$ ! is the factorial of $x$.

The rest of the paper is organized as follows. Section II describes the system model of the multicell cellular network. A description of the relaying schemes is then presented in

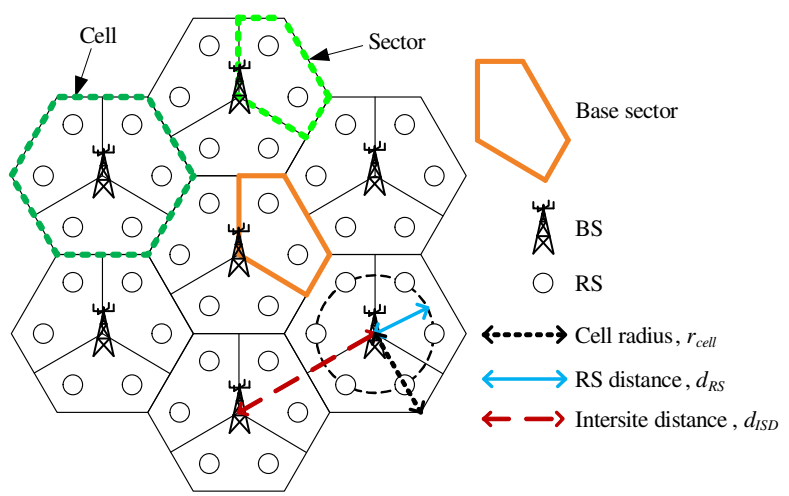

Fig. 1. Topology of the multicell MIMO cellular network with $M=2$ RSs per sector.

Section III, while the user selection methods are outlined in Section IV. In Section V, the cost of the cooperative link is quantified. Some simulation results and discussions are presented in Section VI, while concluding remarks are given in Section VII.

\section{System ModeL}

Let us consider a multicell cellular network shown in Fig. 1 with a 7-cell wrap-around hexagonal structure represented by the set $\mathcal{C}=\{1, \cdots, 7\}$. Each cell is divided into $N_{S e c}$ sectors denoted by the set $\mathcal{S}=\left\{1, \cdots, N_{S e c}\right\}$. In each sector, $M$ equally spaced RSs are positioned at a distance $d_{R S}$ from the cell center, thus forming an arc. The relay set is denoted as $\mathcal{M}=\{1, \cdots, M\}$. A total of $K$ UEs given by the set $\mathcal{K}=\{1, \cdots, K\}$ are uniformly distributed in each sector. We define indexes $b(i, j), r(i, j, m)$ and $u(i, j, k)$ to represent the BS of the $j$ th cell serving the $i$ th sector, the $m$ th RS from the $i$ th sector of the $j$ th cell and the $k$ th user from the $i$ th sector of the $j$ th cell, respectively. It is sufficient to focus on one sector of the central cell as the performance of other sectors is identical on average. We assign $(i, j)=(1,1)$ as the base sector being in focus. Each BS has $N_{b}$ antennas per sector while the number of antennas at the RSs and UEs are $N_{r}$ and $N_{u}$, respectively. The system bandwidth is $B_{\text {sys }}$.

Let $\mathbf{H}_{X, Y} \in \mathbb{C}^{A \times B}$ be the $A \times B$ channel matrix between nodes $X$ and $Y$, where $A$ and $B$ are the number of antennas at $X$ and $Y$, respectively. The elements of $\mathbf{H}_{X, Y}$ are $h_{a, b}=$ $G_{X} \cdot G_{Y} \cdot\left(L_{X, Y}\right)^{-1} \cdot 10^{\frac{\xi_{X, Y}}{10}} \cdot \mu_{X, Y},(1 \leqslant a \leqslant A, 1 \leqslant b \leqslant B)$, where $G_{X}$ and $G_{Y}$ are the transmit antenna gain of $X$ and the receive antenna gain of $Y$, respectively. The path loss between $X$ and $Y$ is $L_{X, Y}$ and is defined in Table I. Next is the log-normal shadowing term with $\xi_{X, Y}$ being a Gaussian distributed random variable having zero mean and standard deviation, $\sigma_{s} \mathrm{~dB}$. Lastly, $\mu_{X, Y}$ denotes the complex Rayleigh fast fading coefficient with unit variance.

\section{A. Downlink transmission protocols}

1) Relay-aided cellular network: For practical reasons, a half-duplex transmission mode is assumed for the RSs since they typically cannot transmit and receive simultaneously on the same time and frequency. Before transmission, the $K$ UEs are assigned into either the direct transmission group denoted 
TABLE I

SIMULATION PARAMETERS.

\begin{tabular}{|c|c|c|}
\hline \multirow{4}{*}{$\begin{array}{l}\text { Path loss, } L_{X, Y} \\
\quad(d \text { in } \mathrm{km})\end{array}$} & BS-RS & $125.2+36.3 \log _{10}(d) \mathrm{dB}$ \\
\hline & BS-UE & $131.1+42.8 \log _{10}(d) \mathrm{dB}$ \\
\hline & RS-UE & $145.4+37.5 \log _{10}(d) \mathrm{dB}$ \\
\hline & RS-RS & $125.2+36.3 \log _{10}(d) \mathrm{dB}$ \\
\hline \multirow{4}{*}{$\begin{array}{l}\text { Shadowing standard } \\
\text { deviation, } \sigma_{s}\end{array}$} & BS-RS & $6 \mathrm{~dB}$ \\
\hline & BS-UE & $10 \mathrm{~dB}$ \\
\hline & RS-UE & $10 \mathrm{~dB}$ \\
\hline & RS-RS & $16.4 \mathrm{~dB}$ shadow margin at $\sigma_{s}=10 \mathrm{~dB}$ \\
\hline \multirow{4}{*}{$\begin{array}{l}\text { Antenna pattern } \\
\left(\theta_{3 \mathrm{~dB}}=70^{\circ}, A_{m}=20 \mathrm{~dB}\right)\end{array}$} & BS & $\rho(\theta)=-\min \left(12\left(\frac{\theta}{\theta_{3 \mathrm{~dB}}}\right)^{2}, A_{m}\right) \mathrm{dB}$ \\
\hline & RS-BS & $\rho(\theta)=-\min \left(12\left(\frac{\theta}{\theta_{3 \mathrm{~dB}}}\right)^{2}, A_{m}\right) \mathrm{dB}$ \\
\hline & RS-UE & Omni \\
\hline & $\mathrm{UE}$ & Omni \\
\hline \multirow{4}{*}{ Antenna gain (boresight) } & BS & $14 \mathrm{dBi}$ (including cable losses) \\
\hline & RS-BS & $7 \mathrm{dBi}$ (including cable losses) \\
\hline & RS-UE & $5 \mathrm{dBi}$ (including cable losses) \\
\hline & $\mathrm{UE}$ & $0 \mathrm{dBi}$ \\
\hline $\begin{array}{c}\text { Noise power } \\
\text { spectral density, } N_{0}\end{array}$ & \multicolumn{2}{|r|}{$-174 \mathrm{dBm}$} \\
\hline Relay time fraction, $\tau_{r}$ & \multicolumn{2}{|r|}{$1 / 2$} \\
\hline Transmit time interval, $T_{T T I}$ & \multicolumn{2}{|r|}{$1 \mathrm{~ms}$} \\
\hline Cooperative time fraction, $\tau_{c o o p}$ & & 0.1 \\
\hline Quantization bits, $\theta$ & \multicolumn{2}{|r|}{4 bits per sample } \\
\hline
\end{tabular}

by set $\mathcal{G}_{\text {Direct }}$ or the relay transmission group denoted by set $\mathcal{G}_{\text {Relay }}$ based on whether direct transmission or relay transmission (using a single RS with the best channel condition) provides better throughput (Fig. 2(a)). The single RS approach is used to avoid high computational complexity at this stage as all the $K$ UEs must be grouped.

In actual relay cooperation transmission using a group of RSs, a user might benefit more from relay transmission rather than direct transmission even though its relay transmission with a single RS performs worse than direct transmission. Nevertheless, the single RS approach is used to avoid the high complexity of sharing the CSI of all the $K$ UEs among all the $M$ RSs at this stage. Thus, we introduce the relay confidence parameter $\beta_{R}\left(0 \leq \beta_{R} \leq 1\right)$ in the transmission group assignment stage to take into account the potential gain of relay cooperation while using the relay transmission with a single RS approach. The direct transmission throughput will be multiplied by a penalizing factor of $\left(1-\beta_{R}\right)$ before being compared to the relay transmission throughput. This allows the performance of conservative and aggressive strategies for allocating users to relay transmission to be studied.

The time division multiple access (TDMA) protocol is utilized for $\mathcal{G}_{\text {Direct }}$ UEs over a duration of $t_{D} T\left(0 \leq t_{D} \leq 1\right)$ where $T$ is the transmission frame interval (Fig. 2(b)). As for the $\mathcal{G}_{\text {Relay }}$ UEs, the two-hop relay transmission protocol is employed as it provides a good tradeoff between performance and complexity. Relay transmission occurs over the remaining time interval of $t_{R} T=T-t_{D} T$ with $0 \leq t_{R} \leq 1$. We select $L$ UEs from $\mathcal{G}_{\text {Relay }}$ for transmission where $L$ is described in Section III. The relay transmission time period $t_{R} T$ is further divided into two time durations. During the first $\tau_{b c} t_{R} T\left(0 \leq \tau_{b c} \leq 1\right)$ duration (broadcast phase), the BS broadcasts the information packets of the $L$ selected UEs to all the $M$ RSs. During the second $\tau_{r} t_{R} T\left(0 \leq \tau_{r} \leq 1\right)$ duration (relay phase), the RSs then relay these packets to the UEs. Here, we define $\tau_{b c}=1-\tau_{r}$ and $t_{R}=\frac{L}{\left|\mathcal{G}_{\text {Direct }}\right|+L}$ where

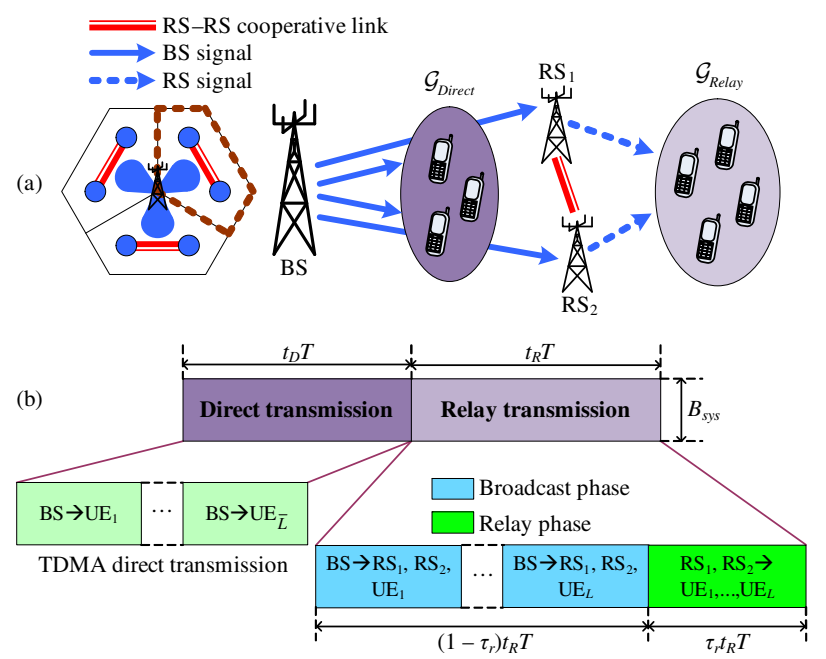

Fig. 2. (a) The relaying structure and (b) the transmission protocol of a relay-aided cellular network employing relay cooperation with $M=2$ RSs per sector.

$\left|\mathcal{G}_{\text {Direct }}\right|+L$ is the the total number of UEs actually selected for transmission during time interval $T$.

2) Direct transmission cellular network: Here, only the BSs participate in data transmission. The BS will transmit directly to the $K$ UEs of each sector in a TDMA arrangement whereby each UE is allocated a transmission duration of $\frac{T}{K}$.

\section{B. Power consumption model}

Let the RF transmit power of the BS be $P_{b}$ per sector. Also, let $P_{r}$ be the RF transmit power allocated to each RS. We employ full relay frequency reuse as it was shown in [26] to be spectrally more efficient when the RS utilizes all sub-channels. In modeling the circuit power consumption, we assume that the circuit power consumption of the BS and RSs is proportional to $P_{b}$ and $P_{r}$, respectively [26]. Let $P_{c, r e f}$ be the circuit power consumption at a given RF transmit power $P_{r e f}$. Therefore, the circuit power consumption of the BS and RS is defined as $P_{c, b}=\frac{P_{b} P_{c, r e f}}{P_{r e f}}$ and $P_{c, r}=\frac{P_{r} P_{c, r e f}}{P_{r e f}}$, respectively.

When measuring total power consumption, we consider the operational power of the system which includes both the RF transmit power and the circuit power. Considering the aggregate effect of the duplexer/feeder losses and the efficiency of the antenna/amplifier modules, let the effective operational efficiency of the BS and RS be given as $\alpha_{b}$ and $\alpha_{r}$, respectively, where $\alpha_{b}, \alpha_{r} \geq 1$. Therefore, the operational power per sector of a relay transmission is

$P_{o p, r e l a y}=\left(1-\tau_{r}\right) \alpha_{b} P_{b}+M \tau_{r} \alpha_{r} P_{r}+P_{c, b}+M P_{c, r}$

while the operational power per sector of a direct transmission is given as

$$
P_{o p, \text { direct }}=\alpha_{b} P_{b}+P_{c, b} .
$$

In this work, we assume that the power consumption scaling is the same for both the BS and RS.

A relay-aided cellular network consists of both direct transmission and relay transmission. Thus, the total operational 
power per sector of a relay-aided cellular network is

$$
P_{o p, t o t a l}^{\text {Relay }}=t_{D}\left(P_{o p, \text { direct }}+M P_{c, r}\right)+t_{R} P_{o p, \text { relay }}
$$

while the total operational power per sector of the direct transmission cellular network is

$$
P_{o p, \text { total }}^{\text {Direct }}=P_{\text {op }, \text { direct }} \text {. }
$$

From (1) and (3), we observe that in a relay transmission the circuit power consumption of the $M$ RSs is the additional power cost that must be accommodated. This additional power cost can quickly become substantial in a network architecture that employs many transmission nodes.

Similar to [26], the energy consumption ratio (ECR) is used as a performance metric for the energy efficiency of a system. It is proportional to the ratio of the average total operational power to the average capacity of the system under consideration. Thus, the ECR is

$$
E C R_{\text {sys }}=\frac{\mathbb{E}\left\{P_{\text {op,total }}^{\text {sys }}\right\}}{B_{\text {sys }} \cdot \mathbb{E}\left\{C_{\text {sys }}\right\}}
$$

where $P_{o p, t o t a l}^{\text {sys }}$ can be either (3) or (4) and $C_{\text {sys }}$ is the spectral efficiency of the system under consideration in bits $/ \mathrm{s} / \mathrm{Hz}$. Therefore, the ECR has units of Joules per bit (J/bit).

\section{Interference analysis}

When all BSs are actively transmitting, the set of interference sources $\mathcal{X}$ experienced by the RSs during the broadcast phase of the relay transmission and by the UEs during direct transmission are from the BSs transmitting to all sectors of all cells except the base sector, that is, $\mathcal{X}=\{(i, j) \mid(i, j) \in \mathcal{C} \times \mathcal{S},(i, j) \neq(1,1)\}$. Assuming the interference sources are independent, the interference covariance matrix for joint RS decoding (JDEC) is a block diagonal matrix given as

$$
\mathbf{R}_{B C}^{J D E C}=\operatorname{diag}\left(\mathbf{U}_{m} \mid m=1, \cdots, M\right)
$$

where $\mathbf{U}_{m}=\sum_{(i, j) \in \mathcal{X}} \frac{P_{b}}{N_{b}}\left(\mathbf{H}_{b(i, j), r(1,1, m)} \mathbf{H}_{b(i, j), r(1,1, m)}^{\mathrm{H}}\right)$. For independent RS decoding (IDEC), the interference covariance matrix at the $m$ th $\mathrm{RS}$ is given as

$$
\mathbf{R}_{B C}^{(m)}=\mathbf{U}_{m}
$$

while for the $k$ th UE, the interference covariance matrix is given as

$$
\mathbf{R}_{D}^{(k)}=\sum_{(i, j) \in \mathcal{X}} \frac{P_{b}}{N_{b}}\left(\mathbf{H}_{b(i, j), u(1,1, k)} \mathbf{H}_{b(i, j), u(1,1, k)}^{\mathrm{H}}\right) .
$$

When all the RSs are actively transmitting, the RSs interfering with the $k$ th UE which is receiving at frequency $f_{u(1,1, k)}$ are the surrounding RSs other than the base sector that are relaying at frequency $f_{r(i, j, m)}$ equal to $f_{u(1,1, k)}$. Thus, the set of RSs interfering the $k$ th UE is $\mathcal{P}_{u(1,1, k)}=$ $\left\{(i, j, m) \mid(i, j, m) \in \mathcal{X} \times \mathcal{M}, f_{r(i, j, m)}=f_{u(1,1, k)}\right\}$. Following that, the interference covariance matrix at the $k$ th UE is

$\mathbf{R}_{R}^{(k)}=\sum_{(i, j, m) \in \mathcal{P}_{u(1,1, k)}} \frac{P_{r}}{N_{r}}\left(\mathbf{H}_{r(i, j, m), u(1,1, k)} \mathbf{H}_{r(i, j, m), u(1,1, k)}^{\mathrm{H}}\right)$

\section{OVERVIEW OF RELAYING TECHNiQues}

We now describe the relaying functions at both the broadcast and relay phases. We utilize the decode-and-forward mechanism whereby the RSs will attempt to decode the received signals before relaying them to the selected UEs. The focus is largely at the relay phase where our proposed relay cooperation technique is implemented. Two other key relaying techniques with contrasting packet forwarding paradigms are also described. For compactness, the index $(i, j)=(1,1)$ is dropped henceforth from expressions containing it as the techniques are explained in relation to the base sector designated as $(1,1)$. For example, the channel matrix $\mathbf{H}_{b(1,1), r(1,1, m)}$ now becomes $\mathbf{H}_{b, r(m)}$. Each RS is assumed to only know the CSI between itself and all the UEs in $\mathcal{G}_{\text {Relay }}$. All other forms of CSI information must be exchanged with other RSs.

\section{A. Broadcast phase: Relay decoding techniques}

1) Joint RS decoding (JDEC): In this strategy, each RS will share its received signals and CSI with all the other RSs through the cooperative links so that they are able to perform joint decoding for all the $L$ selected UE signals. The expressions for $L$ will be described in Sections III-B and III-C. The cooperative links can be a reliable wireless conference channel that utilize a different bandwidth to the underlying cellular network. The cooperative cost of this strategy will be addressed in Section V. Let us define the concatenated broadcast channel matrix, $\mathbf{G}=\left[\mathbf{H}_{b, r(1)}^{\mathrm{T}} \cdots \mathbf{H}_{b, r(M)}^{\mathrm{T}}\right]^{\mathrm{T}}$. Assuming that the CSIs do not change during one transmission frame, the achievable spectral efficiency at the source-relay link for the JDEC strategy is given as

$$
\begin{aligned}
C_{B C, J D E C}= & \left(1-\tau_{r}\right) t_{R} \log _{2} \operatorname{det}\left[\mathbf{I}_{M N_{r}}+\frac{P_{b}}{N_{b}}\right. \\
& \left.\times \mathbf{G G}^{\mathrm{H}}\left(\mathbf{R}_{B C}^{J D E C}+N_{0} B_{s y s} \mathbf{I}_{M N_{r}}\right)^{-1}\right]
\end{aligned}
$$

where the interference covariance matrix $\mathbf{R}_{B C}^{J D E C}$ is defined in (6).

2) Independent RS decoding (IDEC): In this strategy, the RSs will not share their received signals and CSI values to keep complexity low. However, they will still attempt to decode the signals of all the $L$ selected UEs. For successful decoding, the transmission rate of each received signal will be constrained to the minimum spectral efficiency among all $M$ RSs that attempt to decode it. Thus, the achievable spectral efficiency at the source-relay link is

$$
\begin{aligned}
C_{B C, I D E C}^{a l l} & =\min \left\{( 1 - \tau _ { r } ) t _ { R } \operatorname { l o g } _ { 2 } \operatorname { d e t } \left[\mathbf{I}_{N_{r}}+\frac{P_{b}}{N_{b}} \mathbf{H}_{b, r(m)}\right.\right. \\
& \left.\left.\times \mathbf{H}_{b, r(m)}^{\mathrm{H}}\left(\mathbf{R}_{B C}^{(m)}+N_{0} B_{s y s} \mathbf{I}_{N_{r}}\right)^{-1}\right] ; \forall m \in \mathcal{M}\right\}
\end{aligned}
$$

where the interference covariance matrix $\mathbf{R}_{B C}^{(m)}$ is given in (7).

On the other hand, each RS can choose to only decode the signals of the selected UEs that it is assigned to. Let $L_{m}$ be the 
number of selected UEs for the $m$ th RS where $\sum_{m=1}^{M} L_{m}=L$. The achievable spectral efficiency at the source-relay link is, alternatively, given as

$$
\begin{aligned}
C_{B C, I D E C}^{\text {select }}= & \frac{1}{L} \sum_{m=1}^{M} L_{m}\left(1-\tau_{r}\right) t_{R} \log _{2} \operatorname{det}\left[\mathbf{I}_{N_{r}}+\frac{P_{b}}{N_{b}}\right. \\
& \left.\times \mathbf{H}_{b, r(m)} \mathbf{H}_{b, r(m)}^{\mathrm{H}}\left(\mathbf{R}_{B C}^{(m)}+N_{0} B_{s y s} \mathbf{I}_{N_{r}}\right)^{-1}\right] .
\end{aligned}
$$

\section{B. Relay phase: Cooperative multi-processing (CMP) relaying}

In this section, we describe our proposed relay cooperation technique which is an improvement over existing relaying techniques. Here, the RSs will occupy the same relay slot and cooperatively relay the signals received during the broadcast phase to achieve higher spatial multiplexing gain while mitigating multiuser interference, thus, providing higher spectral efficiency improvement over other relaying techniques. Let $L_{C}$ be the number of UEs than can be supported in CMP relaying. The RSs will design the precoder matrix of the $l$ th UE so that its signal is relayed in the null spaces of the remaining $L_{C}-1$ UEs. Thus, the total spatial dimensions of any $L_{C}-1$ UEs must be less than that of the RSs, that is,

$$
\begin{aligned}
\left(L_{C}-1\right) N_{u}<M N_{r} & \Rightarrow L_{C}<\frac{M N_{r}}{N_{u}}+1 \\
& \Rightarrow L_{C}^{*}=\left\lceil\frac{M N_{r}}{N_{u}}\right\rceil
\end{aligned}
$$

where $L_{C}^{*}$ denotes the smallest integer not less than $\frac{M N_{r}}{N_{u}}$ and satisfies the inequality in (13). Considering the actual UEs available in $\mathcal{G}_{\text {Relay }}$, the $L_{C M P}$ UEs effectively selected is given by

$$
L_{C M P}=\min \left\{L_{C}^{*},\left|\mathcal{G}_{\text {Relay }}\right|\right\} .
$$

From (13), we see that each RS selects the total UEs based on the assumption that $M N_{r}$ antennas are available for cooperative relaying, thus, increasing the number of UEs that can be served at a time. The user selection methods for the $L_{C M P}$ UEs will be presented in Section IV.

Let $\mathcal{U}^{C M P}=\left\{u(l) \mid u(l) \in \mathcal{G}_{\text {Relay }}, l=1, \cdots, L_{C M P}\right\}$ be the set of $L_{C M P}$ selected UEs. Their CSIs between each RS are shared among all the RSs. The cooperative cost incurred from this step is investigated in Section V. Also define the concatenated relay channel matrix as seen by the $u(l)$ UE as $\mathbf{F}_{u(l)}=\left[\mathbf{H}_{r(1), u(l)} \ldots \mathbf{H}_{r(M), u(l)}\right]$. The RSs jointly calculate the precoder matrix $\mathbf{W}_{u(l)}$ for $l=1, \cdots, L_{C M P}$, to maximize the relay-destination link spectral efficiency of

$$
\begin{aligned}
C_{R, C M P}= & \max \sum_{u(l) \in \mathcal{U}^{C M P}} \bar{\tau} \log _{2} \operatorname{det}\left[\mathbf{I}_{N_{u}}+M P_{r} \mathbf{F}_{u(l)} \mathbf{W}_{u(l)}\right. \\
& \left.\times \mathbf{W}_{u(l)}^{\mathrm{H}} \mathbf{F}_{u(l)}^{\mathrm{H}}\left(\mathbf{R}_{R}^{u(l)}+N_{0} B_{s y s} \mathbf{I}_{N_{u}}\right)^{-1}\right]
\end{aligned}
$$

where $\bar{\tau}=\tau_{r} t_{R}$ and $\mathbf{R}_{R}^{u(l)}$ is defined in (9). To ensure no multiuser interference, we must have $\mathbf{F}_{u(k)} \mathbf{W}_{u(l)}=\mathbf{0}$ for all $k=1, \cdots, L_{C M P}$ where $k \neq l$. The solution for
$\mathbf{W}_{u(l)}$ that will maximize the spectral efficiency of (15) and simultaneously suppress multiuser interference is obtained through a combination of the singular vector decomposition (SVD) and water-filling approaches. Define $\tilde{\mathbf{F}}_{u(l)}=$ $\left[\mathbf{F}_{u(1)}^{\mathrm{T}} \cdots \mathbf{F}_{u(l-1)}^{\mathrm{T}} \mathbf{F}_{u(l+1)}^{\mathrm{T}} \cdots \mathbf{F}_{u\left(L_{C M P}\right)}^{\mathrm{T}}\right]_{\tilde{\sim}}^{\mathrm{T}}$. First, we obtain the right singular null space vectors of $\tilde{\mathbf{F}}_{u(l)}$, denoted by the column vectors of matrix $\tilde{\mathbf{V}}_{u(l)}^{\text {null }}$. Next, the $r$ singular values of $\left(\mathbf{F}_{u(l)} \tilde{\mathbf{V}}_{u(l)}^{\text {null }}\right)$ are extracted and represented as the diagonals of the $r \times r$ diagonal matrix, $\boldsymbol{\Gamma}_{u(l)}$. Here, $r$ is the rank of $\left(\mathbf{F}_{u(l)} \tilde{\mathbf{V}}_{u(l)}^{\text {null }}\right)$. The right singular vectors corresponding to the $r$ singular values are then found and denoted as the column vectors of matrix $\mathbf{V}_{u(l)}^{\text {base }}$. Next, water-filling is carried out based on $\boldsymbol{\Gamma}_{u(l)}$ to obtain the diagonal power loading matrix $\boldsymbol{\Pi}_{u(l)}$. Finally, the precoder matrix for the $l$ th UE is given as $\mathbf{W}_{u(l)}=\tilde{\mathbf{V}}_{u(l)}^{n u l l} \mathbf{V}_{u(l)}^{\text {base }}\left(\boldsymbol{\Pi}_{u(l)}\right)^{\frac{1}{2}}$. Thus, the overall spectral efficiency of the relay cooperation is

$$
C_{C M P}= \begin{cases}\min \left\{C_{B C, J D E C}, C_{R, C M P}\right\} & \text { if JDEC, } \\ \min \left\{C_{B C, I D E C}^{\text {all }}, C_{R, C M P}\right\} & \text { if IDEC. }\end{cases}
$$

\section{Other relay phase techniques}

1) Interference free (IF) relaying: The "cause no harm" altruistic policy is the principle of this relaying technique. During relay transmission, each RS transmits to its UE group while nulling its transmission towards the other UE groups of the other RSs [28]. Therefore, cooperative information relating to the currently served members in each UE group needs to be exchanged among the RSs. Define $K_{m}$ as the number of UEs assigned to the $m$ th $\mathrm{RS}$ where $\sum_{m=1}^{M} K_{m}=\left|\mathcal{G}_{\text {Relay }}\right|$. Furthermore, let $L_{I}$ be the number of UEs that each RS can support in IF relaying. Each RS must reserve $(M-1) L_{I} N_{u}$ of its spatial dimensions to null interference towards the other UE groups. The remaining $N_{r}-(M-1) L_{I} N_{u}$ spatial dimensions are used to transmit interference free information to its intended $L_{I}$ UEs. To achieve this, the total spatial dimensions of any $L_{I}-1$ intended UEs must be less than the available total spatial dimensions. Thus,

$$
\begin{aligned}
&\left(L_{I}-1\right) N_{u}<N_{r}-(M-1) L_{I} N_{u} \Rightarrow L_{I}<\frac{N r+N u}{M N_{u}} \\
& \Rightarrow L_{I}^{*}=\left\lceil\frac{N_{r}+N_{u}}{M N_{u}}\right\rceil-1
\end{aligned}
$$

where $L_{I}^{*}$ denotes the smallest integer not less than $\frac{N r+N u}{M N_{u}}$ minus one and satisfies the inequality in (17). Considering the actual $K_{m}$ UEs that are available, the $L_{I F, m}$ UEs effectively selected by the $m$ th RS is given by

$$
L_{I F, m}=\min \left\{L_{I}^{*}, K_{m}\right\} .
$$

As each RS must have sufficient spatial dimensions to null interference towards other UEs while still being able to relay useful information to its own UEs, the total number of UEs served by all the $M \mathrm{RSs}$ will be less than that of the proposed CMP relaying technique in Section III-B. Let the set of $L_{I F, m}$ selected UEs by the $m$ th $\mathrm{RS}$ be $\mathcal{U}_{m}^{I F}=\left\{u(l) \mid u(l) \in \mathcal{G}_{\text {Relay }}, l=1, \cdots, L_{I F, m}\right\}$. Thus, the 
relay-destination link spectral efficiency of this relay technique is written as

$$
\begin{aligned}
C_{R, I F} & =\sum_{m \in \mathcal{M}} \sum_{u(l) \in \mathcal{U}_{m}^{I F}} \bar{\tau} \log _{2} \operatorname{det}\left[\mathbf{I}_{N_{u}}+\frac{P_{r}}{L_{I F, m}} \mathbf{H}_{r(m), u(l)}\right. \\
& \left.\times \mathbf{W}_{u(l), m} \mathbf{W}_{u(l), m}^{\mathrm{H}} \mathbf{H}_{r(m), u(l)}^{\mathrm{H}}\left(\mathbf{R}_{R}^{u(l)}+N_{0} B_{s y s} \mathbf{I}_{N_{u}}\right)^{-1}\right]
\end{aligned}
$$

where $\mathbf{W}_{u(l), m}$ is the $m$ th RS precoder of the $l$ th UE. The design of $\mathbf{W}_{u(l), m}$ was given in [28] for a multiple-inputsingle-output (MISO) system. We extend it here to a MIMO system as follows. Let us define the group of other UEs not served by the $m$ th RS as the set given as $\overline{\mathcal{U}}_{m}^{I F}=$ $\left\{\cup \mathcal{U}_{n}^{I F} \mid n \neq m, n \in \mathcal{M}\right\}=\left\{\bar{u}(1), \cdots, \bar{u}\left(\left|\overline{\mathcal{U}}_{m}^{I F}\right|\right)\right\}$. By the SVD, the right singular null space vectors of the vertically stacked channel matrix $\left[\mathbf{H}_{r(m), \bar{u}(1)}^{\mathrm{T}} \cdots \mathbf{H}_{r(m), \bar{u}}^{\mathrm{T}}\left(\left|\overline{\mathcal{U}}_{m}^{I F}\right|\right)\right]^{\mathrm{T}}$ between the $m$ th RS and the UEs in set $\overline{\mathcal{U}}_{m}^{I F}$ is obtained as the column vectors of matrix $\tilde{\mathbf{V}}_{\overline{\mathcal{U}}_{m}^{I F}}^{\text {null }}$. Next, the concatenated channel between the $m$ th RS and the intended UEs in set $\mathcal{U}_{m}^{I F}$ is obtained as $\mathbf{J}_{m}=\left[\mathbf{H}_{r(m), u(1)}^{\mathrm{T}} \cdots \mathbf{H}_{r(m), u\left(L_{I F, m}\right)}^{\mathrm{T}}\right]^{\mathrm{T}}$. A similar procedure as in Section III-B is then applied to the product of $\mathbf{J}_{m} \tilde{\mathbf{V}}_{\overline{\mathcal{H}}_{m}^{\text {IF }}}^{\text {null }}$ for the $m$ th RS instead of jointly for all the $M$ RSs to obtain $\tilde{\mathbf{V}}_{u(l), m}^{\text {null }}, \mathbf{V}_{u(l), m}^{\text {base }}$ and $\left(\boldsymbol{\Pi}_{u(l), m}\right)^{\frac{1}{2}}$. Therefore, the precoder matrix of the $m$ th RS for the lth UE is $\mathbf{W}_{u(l), m}=\tilde{\mathbf{V}}_{\overline{\mathcal{U}}_{m}^{I F}}^{\text {null }} \tilde{\mathbf{V}}_{u(l), m}^{\text {null }} \mathbf{V}_{u(l), m}^{\text {base }}\left(\boldsymbol{\Pi}_{u(l), m}\right)^{\frac{1}{2}}$. The overall spectral efficiency of IF relaying is

$$
C_{I F}=\min \left\{C_{B C, I D E C}^{\text {select }}, C_{R, I F}\right\} .
$$

2) Maximum ratio transmit (MT) relaying: In contrast, each RS in this technique selfishly relays to its associated UE group independent of the other RSs [28]. Each RS performs a maximum ratio transmit precoding on its signals before relaying them to its designated UEs to maximise its own spectral efficiency and to remove multiuser interference from its own UE group. Since it is not coordinated with the other RSs, the receive signal of its UE group will be interfered with by the transmission of the other RSs. Let $L_{M}$ be the number of UEs that each RS can support in MT relaying. As the RSs do not need to null interference to other UE groups, all spatial dimensions can be used to transmit to their own UE groups. Each RS designs the precoder matrix of the lth UE so that its signal is relayed in the null spaces of the remaining $L_{M}-1$ UEs in its group. Therefore, the total spatial dimensions of any $L_{M}-1$ UEs must be less than the available spatial dimensions of the RS. Thus,

$$
\begin{aligned}
\left(L_{M}-1\right) N_{u}<N_{r} & \Rightarrow L_{M}<\frac{N r}{N_{u}}+1 \\
& \Rightarrow L_{M}^{*}=\left\lceil\frac{N r}{N_{u}}\right\rceil
\end{aligned}
$$

where $L_{M}^{*}$ denotes the smallest integer not less than $\frac{N r}{N_{u}}$ and satisfies the inequality in (21). Considering the actual $K_{m}$ UEs (defined above) that are available, the $L_{M T, m}$ UEs effectively selected by the $m$ th RS is

$$
L_{M T, m}=\min \left\{L_{M}^{*}, K_{m}\right\} .
$$

From (17) and (21), we observe that $L_{M}^{*}>L_{I}^{*}$ as the RSs in the MT relaying technique do not need to reserve some of their spatial dimensions for interference suppression but by doing so, incur an interference penalty to the other UEs not in its own relay group. From (13) and (21), we see that $M L_{M}^{*}=$ $L_{C}^{*}$ but the CMP relaying has a further advantage of being able to transmit without interference to the other UEs. Let the set $\mathcal{U}_{m}^{M T}=\left\{u(l) \mid u(l) \in \mathcal{G}_{\text {Relay }}, l=1, \cdots, L_{M T, m}\right\}$ be the selected $L_{M T, m}$ UEs of the $m$ th RS. The relay-destination link spectral efficiency is thus

$$
\begin{aligned}
C_{R, M T}= & \sum_{m \in \mathcal{M}} \sum_{u(l) \in \mathcal{U}_{m}^{M T}} \bar{\tau} \log _{2} \operatorname{det}\left[\mathbf{I}_{N_{u}}+\frac{P_{r}}{L_{M T, m}} \mathbf{H}_{r(m), u(l)}\right. \\
& \times \mathbf{W}_{u(l), m} \mathbf{W}_{u(l), m}^{\mathrm{H}} \mathbf{H}_{r(m), u(l)}^{\mathrm{H}}\left(\sum_{n \in \mathcal{M}, n \neq m} \mathbf{R}_{u(l), n}\right. \\
& \left.\left.+\mathbf{R}_{R}^{u(l)}+N_{0} B_{s y s} \mathbf{I}_{N_{u}}\right)^{-1}\right]
\end{aligned}
$$

where $\mathbf{W}_{u(l), m}$ is the $m$ th RS precoder of the $l$ th UE and

$\sum_{n \in \mathcal{M}, n \neq m} \mathbf{R}_{u(l), n}$ is the interference covariance matrix of the other RSs to the $l$ th UE of the $m$ th RS. Likewise, the design of $\mathbf{W}_{u(l)}$ is described in [28] for a MISO system while we extend it here to a MIMO system. The $m$ th RS calculates the precoder matrix by performing the SVD on the concatenated channel matrix $\mathbf{J}_{m}$ as defined in Section III-C1 but with $u(l) \in \mathcal{U}_{m}^{M T}$ instead. Likewise, a similar procedure as in Section III-B is then applied to $\mathbf{J}_{m}$ to obtain $\tilde{\mathbf{V}}_{u(l), m}^{\text {null }}, \mathbf{V}_{u(l), m}^{\text {base }}$ and $\left(\boldsymbol{\Pi}_{u(l), m}\right)^{\frac{1}{2}}$ for the $m$ th RS instead of jointly for all the $M$ RSs. The precoder matrix of the $m$ th RS for the $l$ th UE is thus $\mathbf{W}_{u(l), m}=\tilde{\mathbf{V}}_{u(l), m}^{\text {null }} \mathbf{V}_{u(l), m}^{\text {base }}\left(\mathbf{\Pi}_{u(l), m}\right)^{\frac{1}{2}}$. The overall spectral efficiency of MT relaying is

$$
C_{M T}=\min \left\{C_{B C, I D E C}^{\text {select }}, C_{R, M T}\right\} .
$$

3) Localized Precoding ( $L o P)$ relaying: A variant of CMP relaying, referred to as LoP relaying, is also compared. Similar to CMP relaying, the RSs cooperate in LoP relaying to select common UEs for transmission. Unlike CMP relaying, the RSs in LoP relaying then transmit data independently to these UEs. Therefore, LoP relaying has limited RS cooperation and is used to illustrate the intermediate change in performance as one evolves from MT relaying with no RS cooperation to full RS cooperation techniques like the IF and CMP relaying.

\section{System capacities}

So far, we have described the capacities of the various types of relay transmission techniques for the UEs in $\mathcal{G}_{\text {Relay }}$ of the relay-aided cellular network. As for the UEs in $\mathcal{G}_{\text {Direct }}$, the TDMA transmission technique is used to transmit information to them. Letting $\bar{L}=\left|\mathcal{G}_{\text {Direct }}\right|$, the achievable spectral efficiency for the UEs in $\mathcal{G}_{\text {Direct }}$ is given by

$$
\begin{aligned}
C_{D}= & \sum_{u(l) \in \mathcal{G}_{\text {Direct }}} \frac{t_{D}}{\bar{L}} \log _{2} \operatorname{det}\left[\mathbf{I}_{N_{r}}+\frac{P_{b}}{N_{b}} \mathbf{H}_{b, u(l)}\right. \\
& \left.\times \mathbf{H}_{b, u(l)}^{\mathrm{H}}\left(\mathbf{R}_{D}^{u(l)}+N_{0} B_{\text {sys }} \mathbf{I}_{N_{r}}\right)^{-1}\right]
\end{aligned}
$$


where the interference covariance matrix $\mathbf{R}_{D}^{u(l)}$ is defined in (8). Therefore, the system spectral efficiency for the relayaided cellular network is give by

$$
C_{\text {relay }}^{(\Theta, \Delta)}=C_{D}+C_{\Theta}[\Delta]
$$

where $C_{\Theta}$ is either (16), (20) or (24) with $\Theta=$ $\{C M P, I F, M T\}$ while $\Delta$ are the user selection methods to be described in Section IV.

In contrast, all $K$ UEs of the set $\mathcal{K}$ in the direct transmission cellular network will be served using the TDMA transmission approach. Its system spectral efficiency is thus

$$
\begin{aligned}
C_{\text {direct }}= & \sum_{u(l) \in \mathcal{K}} \frac{t_{D}}{K} \log _{2} \operatorname{det}\left[\mathbf{I}_{N_{r}}+\frac{P_{b}}{N_{b}} \mathbf{H}_{b, u(l)}\right. \\
& \left.\times \mathbf{H}_{b, u(l)}^{\mathrm{H}}\left(\mathbf{R}_{D}^{u(l)}+N_{0} B_{\text {sys }} \mathbf{I}_{N_{r}}\right)^{-1}\right] .
\end{aligned}
$$

The direct transmission cellular network will be used as a baseline comparison to the various relaying techniques of the relay-aided cellular network.

\section{E. Degree of freedom}

Let us assume there are $L$ UEs participating in the relay transmission. Additionally, let $L_{m}$ be the total UEs assigned to the $m$ th RS for transmission, where $\sum_{m=1}^{M} L_{m}=L$. This is applicable to IF and MT relaying where each RS is assigned to a group of UEs. The total degrees of freedom for CMP, IF and MT relaying can be derived from (13), (17) and (21), respectively, while that of LoP relaying is similar to CMP relaying but without $M$ as the RSs transmit independently. Thus, the total degrees of freedom of the various relaying techniques is given as

$$
\begin{gathered}
\Phi_{C M P}=L \cdot \min \left\{M N_{r}-(L-1) N_{u}, N_{u}, N_{b}\right\}, L \leqslant L_{C}^{*} \\
\Phi_{I F}=L \cdot \min \left\{N_{r}+(1-L) N_{u}, N_{u}, N_{b}\right\}, L \leqslant M L_{I}^{*} \\
\Phi_{M T}=\sum_{m=1}^{M} \min \left\{N_{r}-\left(L_{m}-1\right) N_{u}, N_{u}, N_{b}\right\}, L_{m} \leqslant L_{M}^{*} \\
\Phi_{L o P}=L \cdot \min \left\{N_{r}-(L-1) N_{u}, N_{u}, N_{b}\right\}, L \leqslant L_{M}^{*}
\end{gathered}
$$

where $L_{C}^{*}, L_{I}^{*}$ and $L_{M}^{*}$ are defined in (13), (17) and (21), respectively.

\section{User Selection Methods for Relay COOPERATION}

\section{A. Optimum user selection (OUS)}

In this method, all possible combinations of $L_{C M P}$ UEs in $\mathcal{G}_{\text {Relay }}$ are evaluated and the combination that returns the highest relay-destination link spectral efficiency is selected. The number of combinations is given as

$$
Q=\left(\begin{array}{c}
\left|\mathcal{G}_{\text {Relay }}\right| \\
L_{C M P}
\end{array}\right)=\frac{\left|\mathcal{G}_{\text {Relay }}\right| !}{L_{C M P} !\left(\left|\mathcal{G}_{\text {Relay }}\right|-L_{C M P}\right) !} .
$$

Let set $\mathcal{L}=\left\{\zeta_{q}|q=1, \cdots, Q ;| \zeta_{q} \mid=L_{C M P}\right\}$ contain all the $Q$ possible permutations of the $L_{C M P}$ UEs, each represented by $\zeta_{q}$. Assuming global CSI is available at the RSs, the optimum set of UEs is the one that maximizes (15) and is given as

$$
\mathcal{U}_{O p t}=\underset{\zeta_{q} \in \mathcal{L}}{\arg \max } C_{R, C M P} .
$$

The disadvantage of this method is the cost of exchanging the global CSI and the rapidly increasing cost of computing the $Q$ values of $C_{R, C M P}$ as $\left|\mathcal{G}_{\text {Relay }}\right|$ increases.

\section{B. Full semi-orthogonal user selection (FSUS)}

The OUS method is prohibitive in terms of computational complexity. Given the global CSI, the semi-orthogonal user selection (SUS) method [27] does not need to evaluate all $Q$ permutations. Instead, the UEs are selected sequentially with the channel of each newly added UE being as orthogonal as possible to the channels of the UEs that have already been selected. Thus, computational complexity is reduced albeit with some tolerable performance loss. The SUS idea is incorporated into the FSUS method with the following execution steps.

1) Each RS shares its CSI with all the other RSs.

2) Each RS invokes the SUS algorithm (Fig. 3) using the global CSI in Step 1 as its input. The SUS algorithm is as follows:

a) Firstly, the UE with the highest sum eigenvalues of its RS-UE channel $\mathbf{H}$ is chosen.

b) Next, the UEs that are closely orthogonal to the already selected set of UEs are identified among the remaining UEs.

c) From these UEs, the one with the highest sum eigenvalues is chosen and included into the set of selected UEs.

d) Repeat from Step b) until $L_{C M P}$ UEs are selected. As the global CSI is available, the $L_{C M P}$ selected UEs by each RS will be the same. The disadvantage of the FSUS method is again the cost of exchanging the global CSI.

\section{Partial semi-orthogonal user selection (PSUS)}

The OUS and FSUS methods suffer from costly exchanges of global CSI among the RSs and thus putting a high toll on the cooperative link. Although it is imperative that the RSs must know the CSI for cooperative relaying, the strain on the cooperative link can be decreased by avoiding premature CSI exchange of all the UEs among the RSs. This is the motivation behind the proposed PSUS method with the following execution steps.

1) Each RS independently selects $L_{C M P}$ UEs by invoking the SUS algorithm using its local CSI as input. Consequently, the initially selected UEs may differ from one RS to the other.

2) The $m$ th RS then shares the user index representing its initially selected set of UEs $\mathcal{T}_{m}$ and their corresponding channel norms $\Lambda_{m}$.

3) Using the information at Step 2, each RS invokes the norm-based user negotiation (NUN) algorithm (Fig. 4):

a) The UEs in the first entries of each set $\mathcal{T}_{m}(1)$ are initially selected to include UEs with high channel 


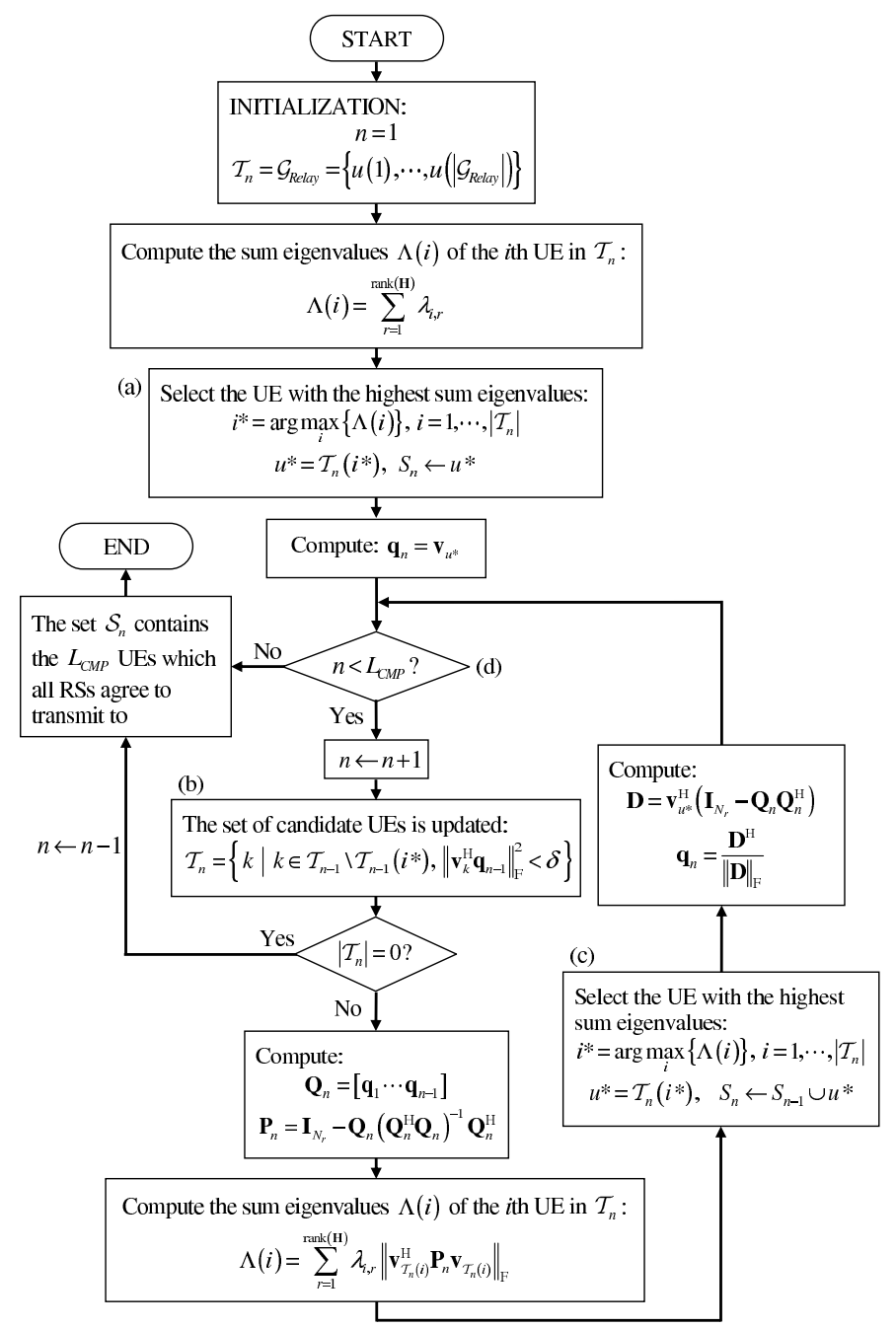

Fig. 3. The SUS algorithm.

norms (UEs are arranged in descending channel norm values).

b) Subsequent UEs in each set are sequentially compared across all sets whereby the UE with the highest channel norm among the currently sequenced UEs is identified.

c) Include this UE into the set of selected UEs only if it is not already selected in the previous selection round.

d) Increment the sequence counter of the selected UE's set.

e) Repeat from Step b) until $L_{C M P}$ UEs are selected.

4) The CSI of only these $L_{C M P}$ UEs which all RSs agree to transmit to are then shared.

The PSUS method reduces the signaling overhead of the cooperative link as it avoids the high cost of communicating the CSI of all the UEs in set $\mathcal{G}_{\text {Relay }}$ to all RSs.

\section{Cooperative Cost}

We assume that the cooperative link has a separate bandwidth $B_{\text {coop }}$ from the underlying cellular system. Let the transmit time interval be $T_{T T I}$ and the fraction of time for cooperative transmission be $\tau_{\text {coop }}\left(0 \leq \tau_{\text {coop }} \leq 1\right)$. At each

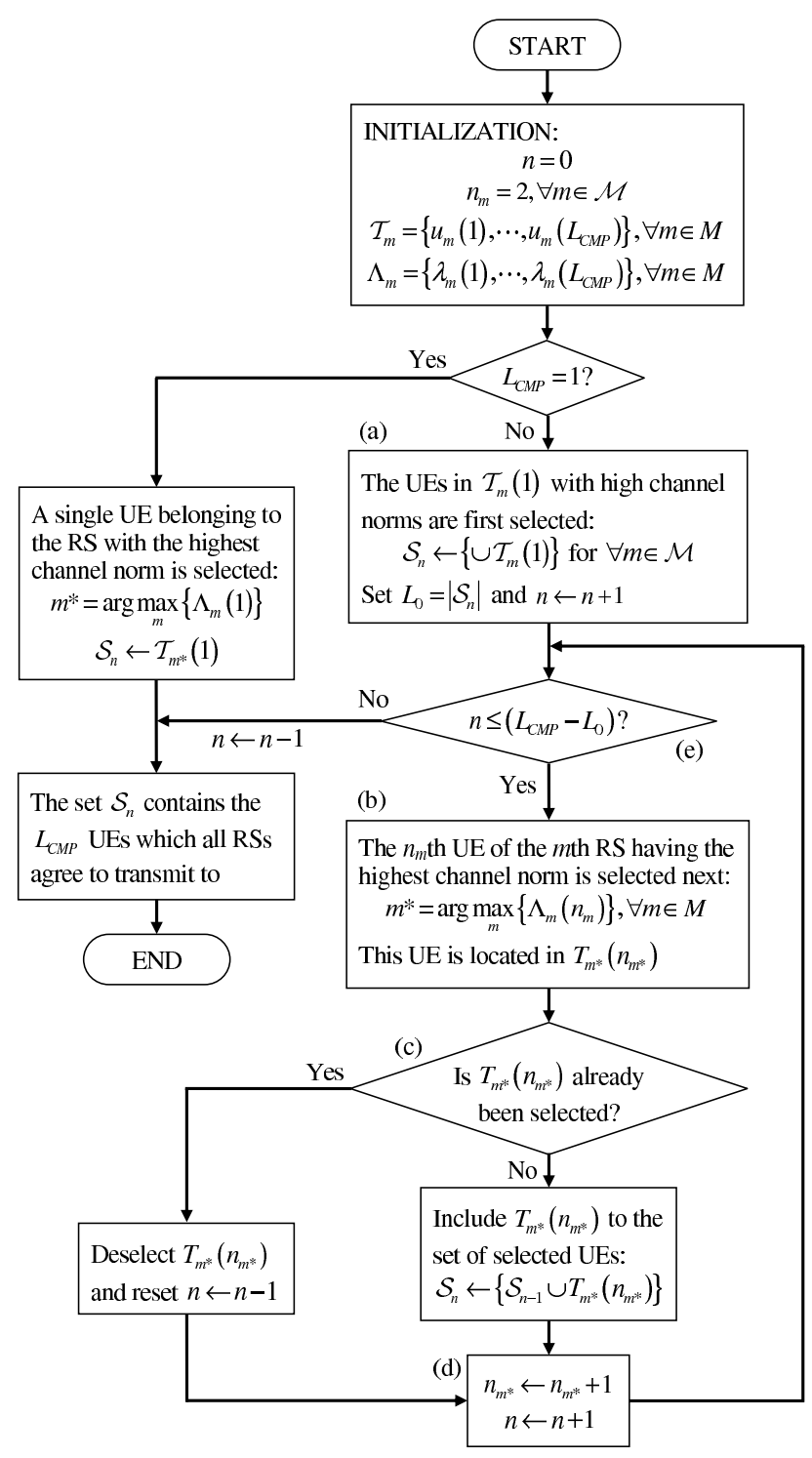

Fig. 4. The NUN algorithm between relays.

RS, the cooperative information will initially be sampled and quantized at a resolution of $\theta$ bits per sample before broadcasting it through the cooperative link to the other RSs.

\section{A. Joint RS decoding (JDEC) cost}

There are two costs associated with the JDEC strategy during the broadcast phase. Firstly, all RSs need to know the CSIs of the other RSs. As we assume that the channel states do not change during one transmission frame, so the CSI needs to be exchanged only once. The second and more costly cooperative information to be frequently exchanged among the RSs are the received signals. All the $M$ RSs must know the other $(M-1)$ RSs received signals each time the BS broadcasts them. We now quantify these two costs.

1) $R S$ received signal exchange cost: For each channel use, the BS transmits a signal vector of length $N_{b}$. Due to the broadcast nature of the BS, each RS will receive a version of this transmit signal as a vector of length $N_{r}$. Since the elements of the received vector are complex numbers with real and imaginary parts, each vector will consist of $2 N_{r}$ 
separate values to be sampled and transmitted through the cooperative link. Assume that the BS will transmit at a rate of $B_{\text {sys }}$ channel uses per second during the broadcast phase, the minimum bit rate that the cooperative link has to support for RS received vector exchanges is given as

$$
R_{J D E C, v e c}=2 N_{r} \theta M B_{\text {sys }} .
$$

Only the CMP relaying technique incurs this cost if it performs JDEC as given in (10) but is exempted from it if IDEC is employed as in (11).

2) Broadcast phase CSI exchange cost: The total elements of the channel matrix between the BS and each RS is $N_{r} N_{b}$. Since the channel matrix elements are complex, there are $2 N_{r} N_{b}$ separate values to be sampled and transmitted through the cooperative link. We assume that the information exchange happens in a fraction $\tau_{\text {coop }}$ of one $T_{T T I}$ duration. Thus, the minimum bit rate that the cooperative link has to support for CSI exchanges during broadcast phase is

$$
R_{J D E C, C S I}=\frac{2 N_{r} N_{b} \theta M}{\tau_{\text {coop }} T_{T T I}} .
$$

Similarly, only the CMP relaying technique incurs this cost if it performs JDEC as given in (10) but is exempted from it if IDEC is employed as in (11).

\section{B. User selection cost}

The CSI exchange which is necessary for the user selection methods occurs once per transmission frame as it is assumed that the channel does not change during that period. Since the user selection methods differ in the extent of the CSI that must be shared, so will the associated costs vary. We will now quantify the cost for the three user selection methods.

1) OUS and FSUS cooperative cost: The total elements of the channel matrix between the $m$ th RS and the $l$ th UE is $N_{u} N_{r}$. Since each channel matrix consists of complex elements, there will be $2 N_{u} N_{r}$ separate values to be sampled and transmitted through the cooperative link for each UE of every RS. In the OUS and FSUS methods, each RS must obtain from the other $(M-1)$ RSs the CSI of all $K$ UEs for its selection mechanism. Thus, assuming that the information is exchanged in a fraction $\tau_{\text {coop }}$ of one $T_{T T I}$ duration, the minimum bit rate that the cooperative link has to support for the OUS and FSUS methods is

$$
R_{O U S}=R_{F S U S}=\frac{2 N_{u} N_{r} K \theta M}{\tau_{\text {coop }} T_{T T I}} .
$$

2) PSUS cooperative cost: Each of the two stages in the PSUS method has an associated cost. After independently selecting the $L_{C M P}$ UEs, each RS broadcasts the channel norms of its selected UEs and an index representing the selected UE sequence. This requires $\left(L_{C M P}+1\right)$ separate values to be sampled and transmitted through the cooperative link for each RS. Next, each RS is required to share the channel matrix between itself and the reselected $L_{C M P}$ common UEs. As the elements of each channel matrix are complex numbers, this requires $2 N_{u} N_{r}$ separate values to be sampled and transmitted through the cooperative link for each reselected UE of every $\mathrm{RS}$. Thus assuming that the information is exchanged in $\tau_{\text {coop }}$ fraction of one TTI duration, the minimum bit rate that the cooperative link has to support for the PSUS method is

$$
R_{P S U S}=\frac{\left(L_{C M P}+1+2 N_{u} N_{r} L_{C M P}\right) \theta M}{\tau_{\text {coop }} T_{T T I}} .
$$

3) Cooperative cost comparison: In order for PSUS to operate at a bit rate that is lower than FSUS, we have

$$
R_{P S U S}<R_{F S U S}
$$

which, after some algebraic manipulation, gives

$$
\begin{aligned}
L_{C M P} & <\frac{2 N_{u} N_{r} K-1}{2 N_{u} N_{r}+1} \\
\Rightarrow \hat{L}_{C M P} & =\left\lceil\frac{2 N_{u} N_{r}(K-1)-2}{2 N_{u} N_{r}+1}\right\rceil
\end{aligned}
$$

where $\hat{L}_{C M P}$ is the largest integer satisfying (39). Therefore, while the $L_{C M P}$ in (14) is necessary due to the dimensional constraint, the $\hat{L}_{C M P}$ in (40) provides a constraint in which PSUS would perform better than FSUS.

\section{Energy efficiency optimization}

We begin by modifying the ECR expression in (5) to include the power consumption of the RS cooperative link. This is given as

$$
\mathrm{ECR}_{\text {sys }}^{\text {modified }}=\frac{\mathbb{E}\left\{P_{\text {op,total }}^{\text {sys }}+P_{o p}^{\text {coop }}\right\}}{B_{\text {sys }} \cdot \mathbb{E}\left\{C_{\text {sys }}\right\}}
$$

where $P_{o p}^{c o o p}$ is the operational power consumption of the RS cooperation link in order to achieve the necessary bit rate of (34)-(37) for cooperative information exchange so that the system could achieve the spectral efficiency of $C_{s y s}$. To minimize (41), we formulate the following problem which is shown for CMP relaying as an example:

$$
\begin{array}{cl}
\underset{N_{r}}{\operatorname{minimize}} & \mathrm{ECR}_{\text {sys, }, M P P}^{\text {modified }} \\
\text { subject to: } & \operatorname{trace}\left(\mathbf{W}_{u(l)} \mathbf{W}_{u(l)}^{\mathrm{H}}\right) \leqslant 1, l=1, \cdots, L_{C M P} \\
& P_{b}, P_{r}>0 \\
& \min \left\{M N_{r}-\left(L_{C M P}-1\right) N_{u}, N_{u}\right\}>0 .
\end{array}
$$

The objective of this constrained optimization problem is to find the optimum number of $\mathrm{RS}$ antennas $N_{r}$ in order to minimize the ECR while considering the cooperative cost. The first constraint ensures that the RS precoder matrix $\mathbf{W}_{u(l)}$ for user $u(l)$ does not violate the power constraint at the RS. The second constraint ensures that the transmit power is always positive. Lastly, the third constraint is the spatial degree of freedom constraint for cooperative relaying among the $M$ RSs.

\section{Simulation Results and Analysis}

We present some numerical results for the downlink transmission of a suburban macrocell scenario. This scenario with a moderately large cell size and medium to heavy traffic load was found to provide the most benefit for relaying techniques. We set $d_{I S D}=1300 \mathrm{~m}$ while $r_{\text {cell }}=d_{I S D} / \sqrt{3}$, $d_{R S}=0.7 r_{\text {cell }}$ and $N_{S e c}=3$. Also, $P_{b}=40 \mathrm{~W}, P_{r}=2 \mathrm{~W}$ and $P_{c, \text { ref }}=577 \mathrm{~W}$ at $P_{\text {ref }}=40 \mathrm{~W}$. Furthermore, we 


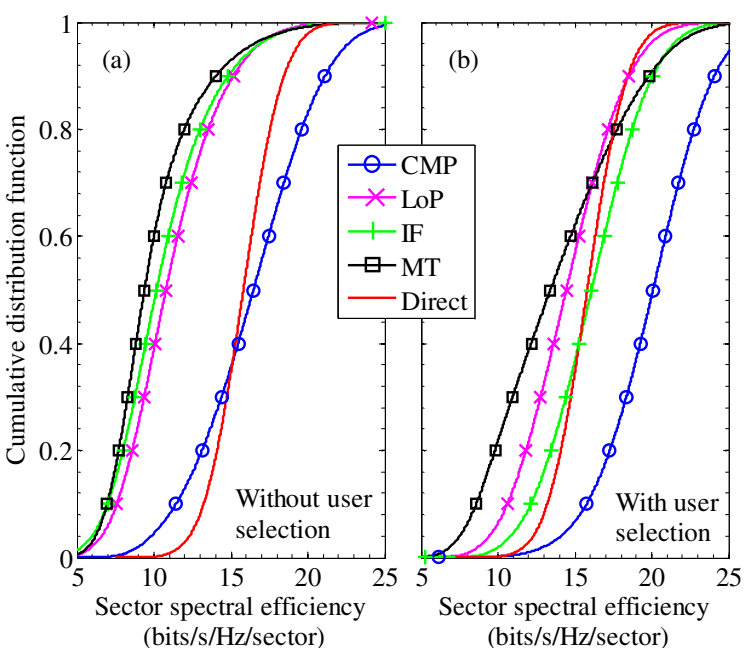

Fig. 5. Link level spectral efficiency of various relaying techniques: (a) without user selection and (b) with the OUS method $\left(N_{b}=8, N_{r}=4\right.$, $\left.N_{u}=2, M=2, K=10\right)$.

set $\alpha_{b}=\alpha_{r}=2.84$ and $B_{\text {sys }}=B_{\text {coop }}=10 \mathrm{MHz}$. The rest of the simulation parameters are in Table I. The link level performance of the CMP, IF, MT and LoP relaying techniques is first evaluated. This is followed by the system level performance evaluation of CMP relaying, considering various combinations of both the JDEC and IDEC strategies with the OUS, FSUS and PSUS user selection methods.

\section{A. Link level performance}

The CMP, IF and MT relaying techniques have different capabilities. For example, both CMP and IF relaying are capable of joint RS decoding during the broadcast phase while MT relaying is not as there is no cooperative link among its RSs. Since the focus of this section is to evaluate the effectiveness of relay phase techniques, IDEC is assumed for all relaying techniques during the broadcast phase. Furthermore, in this subsection, the interference covariance matrix representing the external interference surrounding the base sector is set to zero, leaving only the interference from within the base sector. This setup is to clearly measure the effectiveness of the various relaying techniques in mitigating the interference that is expected from within its own (base) sector. Besides that, the optimum OUS user selection method is employed for all the relaying techniques. This is to ascertain that the performance evaluated is solely on account of the relaying mechanisms during the relay phase alone.

In Fig. 5, the link level spectral efficiency of the relaying techniques is illustrated both with and without the OUS user selection method while direct transmission is taken as the baseline. When no user selection is employed (Fig. 5(a)), the IF relaying performs only slightly better than MT relaying. This is because it has to sacrifice some spatial multiplexing gains for interference free relaying. However, neither performs any better than direct transmission. By contrast, the average spectral efficiency of CMP relaying at $16.5 \mathrm{bits} / \mathrm{s} / \mathrm{Hz} / \mathrm{sector}$ is $5 \%$ better than direct transmission although its spectral efficiency at $10 \%$ outage probability is lower. Despite having access to more spatial dimensions for data transmission, we

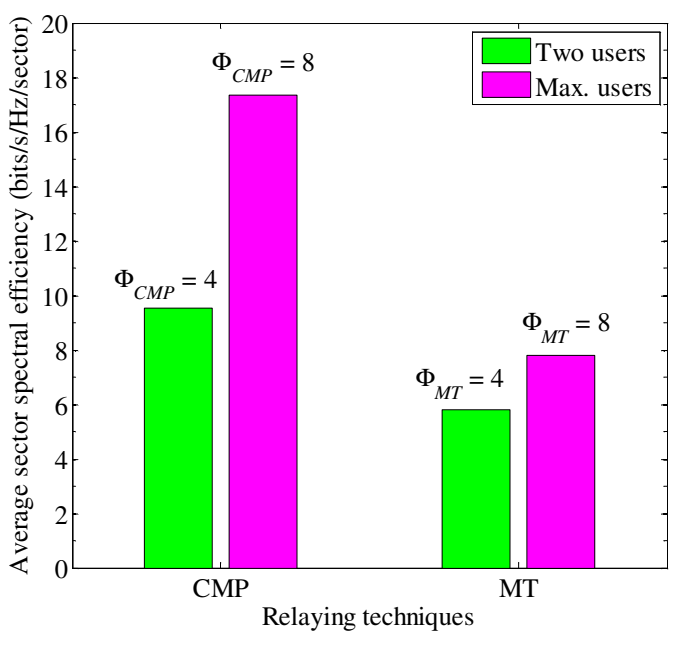

Fig. 6. The spectral efficiency of CMP and MT relaying with two users ( $L=2$ ) where $\Phi_{C M P}=\Phi_{M T}=4$ and with the maximum number of users where $\Phi_{C M P}=\Phi_{M T}=8\left(N_{b}=8, N_{r}=4, N_{u}=2, M=2\right)$.

observe that LoP relaying performs only marginally better than IF relaying which sacrifices some spatial dimension for interference cancellation. The performance loss is due to the signals arriving incoherently at the destinations, resulting in the loss in signal strength.

When user selection is employed in Fig. 5(b), the spectral efficiency of the relaying techniques generally improves due to multiuser diversity. Both MT and IF relaying are now marginally better than direct transmission at higher cumulative distribution function (CDF) values. A clear advantage of IF relaying over MT relaying is now observed. As user selection guarantees that the UEs with the best channel conditions are selected for relay transmission, the channel gain from this outweighs the reduction in spatial multiplexing capability incurred in IF relaying. However, the loss due to incoherent transmission in LoP relaying means that it is not able to benefit from user selection even if the best UEs are selected, resulting in its performance now being poorer than IF relaying. Nevertheless, the benefit of joint relaying transmission in CMP relaying ensures that its spectral efficiency significantly outperforms both the direct transmission and other relaying techniques.

Next, the spectral efficiency of CMP and MT relaying techniques with identical degrees of freedom is compared in Fig. 6. The maximum number of users is when CMP and MT relaying are serving $L_{C}^{*}$ and $M L_{M}^{*}$ UEs, respectively. Going from two users to the maximum number of users, the total degrees of freedom of both CMP and MT relaying increases from 4 to 8 . As the RSs in CMP relaying cooperatively transmit across all spatial dimensions, its spectral efficiency doubles as $\Phi_{C M P}$ doubles. However, as $\Phi_{M T}$ doubles, the spectral efficiency of MT relaying increases by a lesser amount. This is due to the presence of interference at each group of UEs as the RSs in MT relaying transmit independently of one another.

\section{B. System level performance}

We further investigate the performance of CMP relaying at the system level for a relay-aided cellular network where we 


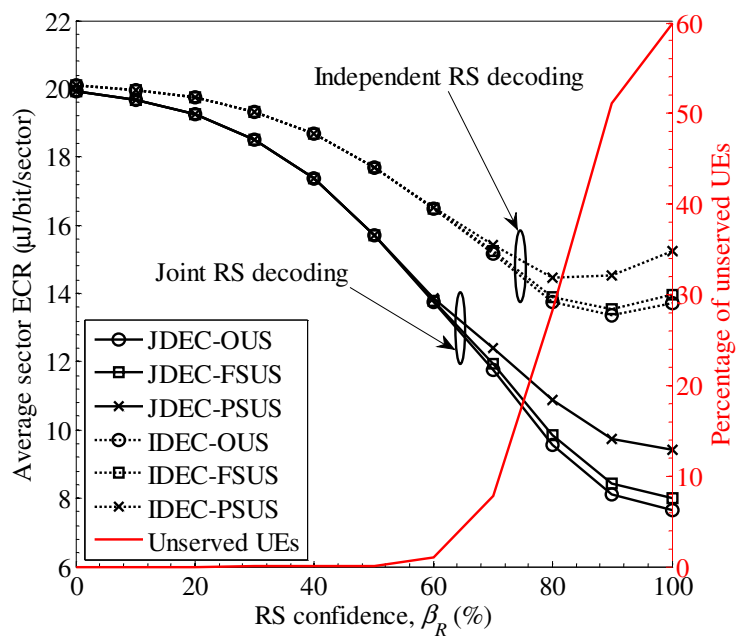

Fig. 7. Average sector ECR and percentage of unserved UEs v.s. relay confidence of CMP relaying $\left(N_{b}=8, N_{r}=4, N_{u}=2, M=2, K=10\right)$.

have both direct and relay transmission present. The effect of external interference surrounding the base sector is considered in this subsection. In Fig. 7, the influence of $\beta_{R}$ on the the ECR performance and percentage of unserved UEs is shown for CMP relaying. At low $\beta_{R}$ (low relay confidence), all UEs will be served by direct transmission ( $0 \%$ unserved UEs) regardless of their distances from the BS. This results in poor energy efficiency as evident from the high ECR values. As $\beta_{R}$ increases (higher relay confidence), more UEs will be assigned to the relay transmission group, especially those further away from the BS. This improves energy efficiency and thus the ECR values decrease. However, due to having the user selection methods, not all of the assigned UEs are selected for relaying and thus the percentage of unserved UEs begins to increase. At $\beta_{R}=1$, all UEs will be assigned to the relay transmission group. From (14), at most $L_{C M P}=4$ UEs will be selected during each cooperative relay transmission, resulting in a maximum of $60 \%$ unserved UEs. To achieve user coverage of around $90 \%$, we therefore select $\beta_{R}=0.7$ as the value for simulation.

In Fig. 8, the spectral efficiency and ECR performance of CMP relaying is evaluated with various BS transmit antennas and different cooperation levels. When JDEC is employed, the broadcast phase is effectively a full spatial multiplexing system with up to $\min \left(N_{b}, M N_{r}\right)$ parallel data streams available between the BS and RSs. Thus, with $N_{r}=4$ and $M=2$, the CMP relaying spectral efficiency in Fig. 8(a) increases as $N_{b}$ increases up to $M N_{r}=8$ antennas. Because $P_{b}$ and $P_{r}$ are fixed, a decrease in ECR is registered in Fig. 8(b) for the same $N_{b}$ range. For $N_{b}>8$, the gains of CMP relaying with JDEC begin to saturate as the number of data streams remains the same. In IDEC, each RS has only its own antennas for decoding, thus, supporting min $\left(N_{b}, N_{r}\right)$ parallel data streams between the BS and each RS. This limits the gains when $N_{b}>4$. A performance gap of around $1.3 \mathrm{bit} / \mathrm{s} / \mathrm{Hz} / \mathrm{sector}$ for spectral efficiency and $3.1 \mu \mathrm{J} / \mathrm{bit} / \mathrm{sector}$ for ECR is typically observed between JDEC and IDEC. However, the superior performance of JDEC requires a prohibitively high cooperative cost, as we shall see later.
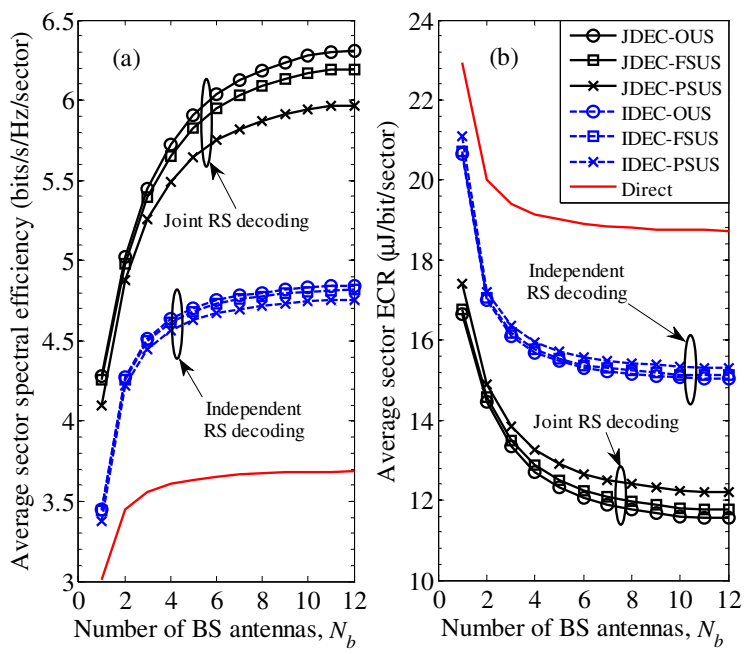

Fig. 8. (a) Spectral efficiency and (b) ECR v.s. the number of BS antennas for CMP relaying in a relay-aided cellular network $\left(\beta_{R}=70 \%, N_{r}=4\right.$, $\left.N_{u}=2, M=2, K=10\right)$.

TABLE II

Cooperative Cost.

\begin{tabular}{|c|c|c|c|c|c|}
\hline \multirow{2}{*}{ Strategies } & \multicolumn{3}{|c|}{$\begin{array}{c}\text { Broadcast phase: } \\
\text { Joint RS decoding (JDEC) }\end{array}$} & \multicolumn{3}{c|}{ User selection method } \\
\cline { 2 - 6 } & Signal sharing & CSI sharing & OUS & FSUS & PSUS \\
\hline Bit rate (Mbits/s) & 640 & 5.12 & 12.8 & 12.8 & 5.52 \\
\hline RF transmit power (mW) & very high & 58.2 & 164.6 & 164.6 & 63.2 \\
\hline Operational power (W) & very high & 1.0 & 2.8 & 2.8 & 1.1 \\
\hline
\end{tabular}

The impact of various user selection methods on the CMP relaying system performance is also evident in Fig. 8. As expected, OUS performs the best while FSUS performs slightly better than PSUS. The performance gap between OUS and PSUS is more significant when JDEC, rather than IDEC, is considered. This is because the performance of CMP relaying with JDEC is limited by the relay phase. Thus, the type of user selection method will influence the overall system performance more profoundly as it is implemented at the relay phase. This is less significant in CMP relaying with IDEC where the bottleneck is at the broadcast phase.

The cooperative costs of CMP relaying for different cooperative levels are tabulated in Table II. It is established that CMP relaying with JDEC performs better than IDEC both in terms of spectral efficiency and energy efficiency. To realize such gains, the cooperative links between the RSs must be able to at least support a bit rate of $640 \mathrm{Mbits} / \mathrm{s}$ to share all the signals received during the broadcast phase. This bit rate is an order of magnitude higher than that can be delivered by the underlying relay-aided cellular system. Besides that, CSI sharing requires a further bit rate of at least $5.12 \mathrm{Mbits} / \mathrm{s}$. Needless to say, the power consumption to operate the cooperative link will be excessively high. Therefore, the performance gain of CMP relaying with JDEC must be traded off against a very large increase in cooperative cost.

From Table II, both the OUS and FSUS methods require a cooperative link with a bit rate of $12.8 \mathrm{Mbits} / \mathrm{s}$. Assuming an $N_{r} \times N_{r}$ MIMO cooperative link with a shadow margin of 16.4 $\mathrm{dB}$ (corresponding to $\sigma_{s}=10 \mathrm{~dB}$ ) for $90 \%$ link reliability, the $\mathrm{RF}$ transmit power and operational power are $164 \mathrm{~mW}$ and 2.8 


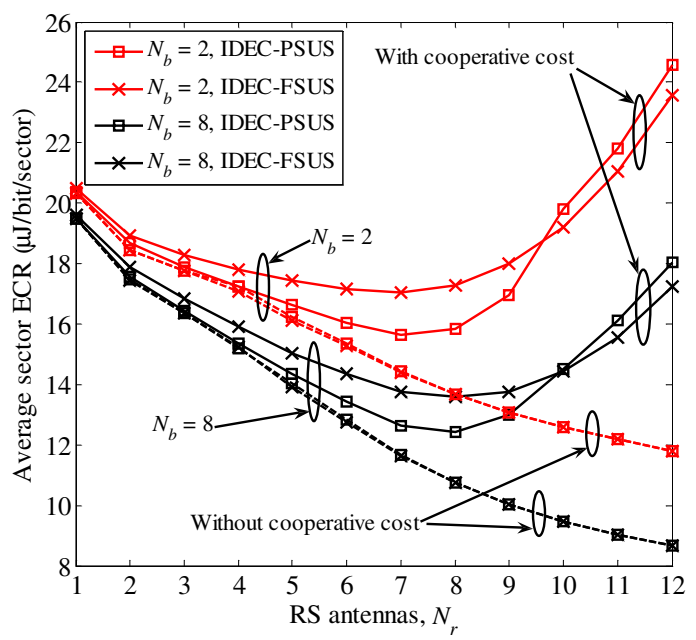

Fig. 9. The average sector ECR v.s. the number of RS antennas of the IDEC-PSUS and IDEC-FSUS relay cooperation schemes for both with and without the cooperative cost for $N_{b}=2$ and $N_{b}=8$.

$\mathrm{W}$, respectively. The operational power is calculated using (2) as direct transmission is assumed for the cooperative links. As for the PSUS method, the cooperative link has to support a bit rate of just $5.52 \mathrm{Mbits} / \mathrm{s}$ and operational power of $1.1 \mathrm{~W}$. Although the PSUS method performs slightly worse than the OUS and FSUS methods in terms of system performances, this shortcoming is more than compensated by being able to operate at less than half the cooperative cost of the other two methods.

In Fig. 9, the ECR of (41) is numerically optimized for IDEC-PSUS and IDEC-FSUS relay cooperation schemes while considering the cooperative cost of two different user selection methods. The ECR of (5) is also evaluated to illustrate the performance when the cooperative cost is not considered. For both IDEC-PSUS and IDEC-FSUS, the ECR with no cooperative cost decreases monotonically with $N_{r}$. Furthermore, for a given $N_{b}$, the ECR performance of PSUS without cooperative cost is slightly worse than FSUS. When the cooperative cost is considered, the minimum ECR is achieved for both schemes when $N_{r}=7$ and $N_{r}=8$ for $N_{b}=2$ and $N_{b}=8$, respectively. It is also observed that PSUS outperforms FSUS by registering lower ECR values when cooperative cost is included. For $N_{r}>8$, the operational power consumption at the RS cooperation link becomes excessively large as the required bit rate for the exchange of cooperative information becomes prohibitively high. This results in increasing ECR values. For $N_{r} \geq 10$, the performance of PSUS begins to degrade as compared to FSUS. This is because at higher $N_{r}$ values, the number of UEs supported for joint transmission, which is given by (14), surpasses the threshold in (40), i.e., $L_{C M P}>\hat{L}_{C M P}$ for $N_{r} \geq 10$. For example, at $N_{r}=11$, we have $L_{C M P}=11$ while $\hat{L}_{C M P}=9$, resulting in PSUS having a higher ECR value than FSUS.

\section{CONCLUSIONS}

A relay cooperation scheme has been proposed for downlink multicell MIMO cellular networks. Different RS decoding strategies (JDEC and IDEC) for the broadcast phase and joint relay transmission with different degrees of CSI sharing for the relay phase have been investigated. It has been demonstrated that compared with direct transmission, relay cooperation can achieve energy reductions of up to $36 \%$ with JDEC and up to $19 \%$ with IDEC. However, JDEC requires a cooperative link with a bit rate of an order of magnitude greater than that achievable by the relay network. We have also proposed the PSUS user selection method for relay phase joint transmission which enables the cooperative link to operate at $39 \%$ of the cooperative cost incurred by competing methods that require global CSI. This significant cost reduction more than compensates for the slight degradation caused by the PSUS method to the system performance.

\section{REFERENCES}

[1] 3GPP, TR36.814, V1.0.1, "3rd Generation Partnership Project; Technical Specification Group Radio Access Network; Further Advancements for E-UTRA Physical Layer Aspects," Sept. 2008.

[2] J. N. Laneman, D. N. C. Tse, and G. W. Wornell, "Cooperative diversity in wireless networks: efficient protocols and outage behaviour," IEEE Trans. Inf. Theory, vol. 50, no. 12, pp. 3062-3080, Dec. 2004.

[3] Q. Lin, X. Wang, Y. Wang, and J. Liao, "Performance evaluation of frequency planning in a novel cellular architecture based on sector relay," in Proc. 2010 IEEE VTC - Spring, pp. 1-5.

[4] S. Kim, W. Choi, Y. Choi, J. Lee, and Y. Han, "Interference reduction of cellular relay networks in multiple-cell environment by spectrum agility," in Proc. 2008 IEEE PIMRC, pp. 1-5.

[5] A. Austin and J. Vidal, "Amplify-and-forward cooperation under interference-limited spatial reuse of the relay slot," IEEE Trans. Wireless Commun., vol. 7, no. 5, pp. 1952-1962, May 2008.

[6] O. Simeone, O. Somekh, Y. Bar-Ness, H. V. Poor, and S. Shamai, "Capacity of linear two-hop mesh networks with rate splitting, decodeand-forward relaying and cooperation," in Proc. 2007 Allerton on Communication, Control, and Computing.

[7] W. Chen, K. B. Letaief, and Z. Cao, "Network interference cancellation," IEEE Trans. Wireless Commun., vol. 8, no. 12, pp. 5982-5995, Dec. 2009.

[8] X. Hong, Y. Jie, C.-X. Wang, J. Shi, and X. Ge, "Energy-spectral efficiency trade-off in virtual MIMO cellular systems," IEEE J. Sel. Areas Commun., vol. 31, no. 10, Oct. 2013.

[9] I. Ku, C.-X. Wang, and J. S. Thompson, "The spectral, energy and economic efficiency of relay-aided cellular networks," IET Commun., accepted for publication.

[10] X. Ge, K. Huang, C.-X. Wang, X. Hong, and X. Yang, "Capacity analysis of a multi-cell multi-antenna cooperative cellular network with co-channel interference," IEEE Trans. Wireless Commun., vol. 10, no. 10, pp. 3298-3309, Oct. 2011.

[11] P. Wang, H. Wang, L. Ping, and X. Lin, "On the capacity of MIMO cellular systems with base station cooperation," IEEE Trans. Wireless Commun., vol. 10, no. 11, pp. 3720-3731, Nov. 2011.

[12] C. Abgrall, E. C. Strinati, and J. -C. Belfiore, "Inter-cell interference mitigation allocation for half-duplex relays based cooperation," in Proc. 2009 IEEE WD, pp. 1-6.

[13] H. Ganapathy, J. G. Andrews, and C. Caramanis, "Inter-cell relay cooperation in heterogeneous cellular uplink systems," in Proc. 2008 ACSSC, pp. 1443-1447.

[14] Y. Kim, T. Kim, S. Kim, and Y. Han, "Adaptive cooperation strategy for multiple relays in 4G wireless systems," in Proc. 2009 IEEE WCNC, pp. $1-5$.

[15] A. W. Eckford, J. P. K. Chu, and R. S. Adve, "Low complexity and fractional coded cooperation for wireless networks," IEEE Trans. Wireless Commun., vol. 7, no. 5, pp. 1917-1929, May 2008.

[16] M. A. Torabi and J. F. Frigon, "A decomposition approach to MIMO interference relay networks," in Proc. 2008 IEEE GLOBECOM, pp. 1-6.

[17] B. Niu and A. M. Haimovich, "Optimal throughput of two-hop relay networks with different relay cooperation," in Proc. 2009 IEEE MILCOM, pp. $1-5$.

[18] M. P. Stef, Z. A. Polgar, and V. Bota, "Network-coded cooperation protocol for multiple source-multiple relay topologies in cellular networks," in Proc. 2010 Future Network and Mobile Summit, pp. 1-9.

[19] W. Zeng, C. Xiao, Y. Wang, and J. Lu, "Opportunistic cooperation for multi-antenna multi-relay networks," IEEE Trans. Wireless Commun., vol. 9, no. 10 , pp. 3189-3199, Oct. 2010. 
[20] J. Zhang and K. B. Letaief, "Interference management with relay cooperation in two-hop interference channels," IEEE Wireless Commun. Lett., vol. 1, no. 3, pp. 165-168, June 2012.

[21] N. Devroye, N. B. Mehta, and A. F. Molisch, "Asymmetric cooperation among wireless relays with linear precoding," IEEE Trans. Wireless Commun., vol. 7, no. 12, pp. 5420-5430, Dec. 2008.

[22] R. Madan, N. B. Mehta, A. F. Molisch, and J. Zhang, "Energy-efficient cooperative relaying over fading channels with simple relay selection," IEEE Trans. Wireless Commun., vol. 7, no. 8, pp. 3013-3025, Aug. 2008.

[23] C. Han, T. Harrold, S. Armour, I. Krikidis, S. Videv, P. M. Grant, H. Haas, J. S. Thompson, I. Ku, C.-X. Wang, T. A. Le, M. R. Nakhai, J. Zhang, and L. Hanzo, "Green radio: radio techniques to enable energy efficient wireless networks," IEEE Commun. Mag., vol. 49, no. 6, pp. 4654, June 2011.

[24] O. Oyman and A. J. Paulraj, "Power-bandwidth tradeoff in dense multiantenna relay networks," IEEE Trans. Wireless Commun., vol. 6, no. 6, pp. 2282-2293, June 2007.

[25] X. Lao, L. Cuthbert, T. Zhang, and L. Xiao, "Energy efficiency and optimal resource allocation in cooperative wireless relay networks," in Proc. 2011 IEEE VTC - Spring, pp. 1-6.

[26] I. Ku, C.-X. Wang, and J. Thompson, "Spectral-energy efficiency tradeoff in multicell cellular networks with adaptive relay cooperation," in Proc. 2012 IEEE GLOBECOM, pp. 4585-4590.

[27] T. Yoo and A. Goldsmith, "On the optimality of multiantenna broadcast scheduling using zero-forcing beamforming," IEEE J. Sel. Areas Commun., vol. 24, no. 3, pp. 528-541, Mar. 2006.

[28] J. Zhou and J. Thompson, "Linear precoding for the downlink of multiple input single output coexisting wireless systems," IET Commun., vol. 2, no. 6, pp. 742-752, Mar. 2008.

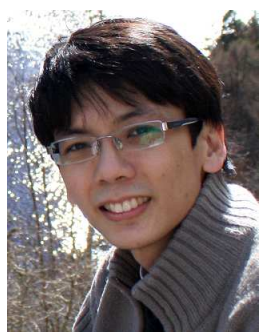

Ivan Ku received the B.Eng. degree in Electronics and M.Eng.Sc. degree in Telecommunications from Multimedia University, Cyberjaya, Malaysia, in 2001 and 2006, respectively, and the Ph.D. degree in Wireless Communications from Heriot-Watt University and the University of Edinburgh, Edinburgh, United Kingdom, in 2013. His research interests include green communications, cooperative MIMO systems, multi-user detection, channel coding, and cognitive radio networks.

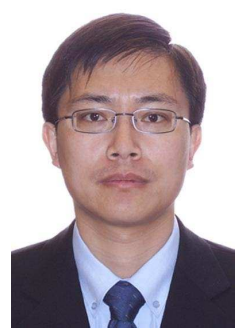

Cheng-Xiang Wang (S'01-M'05-SM'08) received the B.Sc. and M.Eng. degrees in Communication and Information Systems from Shandong University, Shandong, China, in 1997 and 2000, respectively, and the Ph.D. degree in Wireless Communications from Aalborg University, Aalborg, Denmark, in 2004.

Since 2005, he has been with Heriot-Watt University, Edinburgh, U.K., first as a Lecturer, then as a Reader in 2009, and as a Professor in 2011. $\mathrm{He}$ is also an Honorary Fellow of the University of Edinburgh and a Chair/Guest Professor with Shandong University and Southeast University, China. From 2001 to 2005, he was a Research Fellow with the University of Agder, Grimstad, Norway. In 2004, he was a Visiting Researcher with Siemens AG-Mobile Phones, Munich, Germany. From 2000 to 2001, he was a Research Assistant with the Technical University of Hamburg-Harburg, Hamburg, Germany. He is the Editor of one book. He has published one book chapter and over 180 papers in refereed journals and conference proceedings. His research interests include wireless channel modeling and simulation, green communications, cognitive radio networks, vehicular communication networks, large MIMO, cooperative MIMO, and Beyond-Fourth-Generation wireless communications.

Prof. Wang is a Fellow of the Institution of Engineering and Technology and the HEA and a member of the Engineering and Physical Research Council Peer Review College. He has served as an editor for eight international journals, including the IEEE TRANSACTIONS ON VEHICULAR TECHNOLOGY (since 2011) and the IEEE TRANSACTIONS ON WIRELESS COMmunications (2007-2009). He was the lead Guest Editor for the IEEE Journal on Selected Areas in Communications Special Issue on Vehicular Communications and Networks. He has served as a Technical Program Committee (TPC) Member, TPC Chair, and General Chair for more than 70 international conferences. He received the Best Paper Awards at the 2010 IEEE Global Telecommunications Conference, the 13th IEEE International Conference on Communication Technology in 2011, and the 12th International Conference on ITS Telecommunications in 2012.

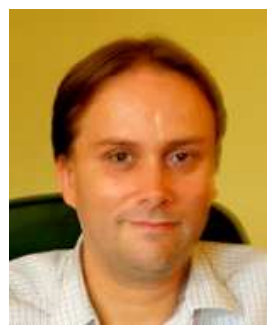

John S. Thompson (M'94-SM'13) received his B.Eng. and Ph.D. degrees in Electronic and Electrical Engineering from the University of Edinburgh in 1992 and 1996, respectively.

He was appointed as a lecturer at what is now the School of Engineering at the University of Edinburgh in 1999. He was recently promoted to a personal chair in Signal Processing and Communications. His research interests currently include signal processing, energy efficient communications systems, and multi-hop wireless communications. He has published over 200 papers to date including a number of invited papers, book chapters and tutorial talks, as well as co-authoring an undergraduate textbook on digital signal processing. During 2012-2014 he is serving as member-at-large for the Board of Governors of the IEEE Communications Society. He was technical programme co-chair for the IEEE Globecom Conference in Miami in 2010 and served recently in the same role for the IEEE Vehicular Technology Conference Spring in Dresden in 2013. 